\title{
Investigating the Equity of Access When Comparing Migrant and Local Students
}

\author{
Jiayi Zhang* \\ Shengli NO.1 Middle school, Dongying, Shandong, China \\ *Corresponding autho Email: guanghua.ren@gecacademy.cn
}

\begin{abstract}
This paper reviews the disadvantageous position of migrant students in urban areas since numerous people hold a wrong notion that migrant students are so fortunate to go to urban schools and accept relatively more developed instructions on schoolwork. However, there are still many barriers for migrant students to have equal access to quality education as local urban students. This paper will investigate external and psychological factors contributing to this situation and illustrate the possible influence on migrant students. First, in terms of external factors, it is common for migrant students to be discriminated against by local citizens. Secondly, some migrant students attending local migrant schools are threatened by the weak teachers. In addition, the absence of parental care in children's growth results in some behavioral problems, and the lack of parental supervision in schoolwork tends to cultivate many unhealthy study habits. Regarding migrant students' psychological condition, they are inclined to have some mental problems. The inferiority complex is a negative mental state of migrant students due to the contrast between rural life standard and urban life standard, and loneliness is mainly caused by insufficient interactions between migrant students and local citizens. Moreover, the identification problem mirrors migrant students' distinct definitions of their identity. Overall, the disadvantages curbs migrant students' integration into the urban society, and in the long-run, their future development.
\end{abstract}

Keywords: Migrant students, Inferior position, Discrimination, Migrant school, Parental support, Inferiority complex, Loneliness, Identification problem.

\section{INTRODUCTION}

Since the 1980s, there have been some modifications in regulations regarding the movement between rural and urban areas. Hence, plenty of rural labor forces have started to move to cities, thereby bringing about the largest population shift in the world [1]. A vast majority of those workers have brought their children to cities, which offers opportunities for those migrant students to live in a relatively more developed environment. However, there are many barriers for those migrant students to have equal access to quality education as urban students. Many previous studies have investigated the causes of the inferior position for migrant students and their effects. The findings can be divided into two main facets in terms of causes, including external factors and psychological states.

\section{MAIN BODY}

\subsection{External Factors}

\subsubsection{Discrimination from the Locals}

Migrant students are exposed to discrimination from local people often. The discrimination prevents migrant students from adapting to the urban environment, a relatively new and unfamiliar environment. Numerous local citizens might not accept migrant workers and their children to live in cities and are against migrant students going to urban state schools [2]. An interview argues that many migrant students lack a sense of belonging in cities due to the stereotype of local people on them [3]. Goodburn finds that the rural accents and dressing styles of migrant students are often greeted with derision in cities [3]. Therefore, it is natural for migrant students to believe that they are not being accepted and understood in urban society, which leads to many mental problems of migrant students. One study refers that the urban 
experiences of migrant students, the formation of proper perspective of themselves, other people, and the whole community and their personality are dependent upon how they are treated by people around them [2].

\subsubsection{The Influence of Migrant Schools}

Migrant schools are schools established specifically for migrant students. Many reasons explain why some migrant students go to migrant schools rather than state schools in the cities. Firstly, choosing to go to migrant schools effectively avoids the need for legal "Hukou" status for attending state schools and high expenditure for tuition fees. "Hukou" is an official document issued by the Chinese government, certifying that the holder is a legal resident of a particular area according to Oxford Dictionary. However, the academic performance of migrant students in migrant schools is highly threatened.

To start with, one significant concern regarding migrant schools is the teaching ability and skill of teachers working in these unlicensed schools due to the fact that a vast majority of teachers do not have sufficient professional knowledge and fundamental skills as teachers [3]. Therefore, the quality of the content of courses offered in migrant schools is much poorer than that received by local students, which leads to poorer academic achievement of migrant students attending migrant schools than that of local students. A survey finds that the grades of urban students are better than the scores of migrant students [4]. The study results indicate that most migrant students' academic performance is below the middle level. Among his sample students, only $10.9 \%$ of the respondents are excellent at learning, and the grades of $37.9 \%$ of the migrant students are below the middle level, whereas $20 \%$ are considered to be quite poor at schoolwork [4].

Also, there are obvious drawbacks of the setup of non-examination courses in migrant schools [3]. Those courses are not academically theoretical subjects such as mathematics and physics, but they are also beneficial for migrant students' comprehensive quality. One survey finds that students can boost their brainpower through the study of music [5]. Liu argues that students' critical thinking can be fostered by accepting practices regarding music since learning music helps students activate the semi-sphere of their brain [5]. Liu also finds that after taking music courses, students tend to be more disciplined and more persistent in schoolwork [5]. In migrant schools, the absence of these courses contributes to the undesirable performance of migrant students in these professional areas. A survey refers that urban students are generally more versatile than migrant students [4].

\subsubsection{The Influence of Family Environment and Parental Support}

The family factor contains several aspects. This section focuses on the effect of communications between parents and migrant students and the school transfer on migrant students. The results of investigations in this field illustrate that the academic and mental development of migrant students is under great influence of this factor.

Firstly, migrant workers are in relatively more disadvantaged positions in comparison with local citizens [6]. Tian found in his survey that the income of migrant workers is generally less than that of urban workers [6]. Hence, the target of enabling their children to enjoy the same standard of living and quality of life as urban children pushes migrant workers to work overtime and have part-time jobs in order to earn more money. This directly results in a lack of time for communication between migrant workers and their children. It can be common for migrant workers not to react to the affection need of their children. Without the basic interactions in families, the relationship between parents and children can be jeopardized considerably. An investigation refers that family plays a pivotal role in moulding the characters of children [7]. Without receiving enough care in families, what migrant students may do is to conduct some misbehavior. From migrant students' perspective, it is more likely for their parents to focus more on them by doing something that exceeds what is proper. It is described in some investigations that more behavioral problems can be seen in migrant students compared with urban students, including internalizing and externalizing problem behaviors [2].

In the second place, migrant workers might not have enough experience and techniques to help their children with their school projects due to the fact that many migrant workers have not accepted any education or have only experienced fundamental levels of education. Owing to heavy workloads, it is also impossible for migrant workers to spare much time to supervise their children on schoolwork and check for their children's homework on time. Therefore, it is very likely for migrant students to take up some bad habits due to the lack of help from parents [4]. For example, the survey done by Xie illustrates that playing computer games is a common habit developed by migrant students. Among the chosen study subjects, some migrant students tend to be addicted to electronic devices [4].

Additionally, school transfers are normal in migrant students' educational experiences owing to the mobility of their parents' jobs. However, going to a new school requires a period of adaptation. For example, migrant students must get to know new students and teachers in their new schools, thereby further integrating into a new social group, which takes time and is also based on their adaptability. Many studies show that the adaptability of 
migrant students is poorer than that of local students [8]. In addition, the anxiety migrant students experience in school projects is more severe than local students [9]. Thus, during the adaptive process, migrant students may not be able to entirely concentrate on study, contributing to low study productiveness and low grades at school.

\subsection{Negative Psychological States}

\subsubsection{Inferiority Complex}

It has been illustrated that migrant students have weaker sense of well-being than urban students due to psychological problems [2]. A typical problem is inferiority complex. A survey argues that a large number of migrant students suffer from the problem of inferiority complex [11]. Sun et al. illustrate that some migrant students believe they are born incompetent with no good qualities [11]. Another investigation finds that nearly one-fourth of the migrant students have inferiority complex problems [12].

One reason to explain this situation of migrant students is that the rural-to-urban shift leaves them with the problem of dealing with new environments. To be more specific, comparing the distinctions between the lifestyle of urban students and that of rural students is a natural reaction in their minds after contacting urban people. As a result, they are inclined to only focus on their disadvantages and block all the other circumstances because of their narrow perspectives, thereby producing a negative mental state of mind - inferiority complex, which may significantly curb the cultivation of the potentials of students. The inferiority complex results in a negative mindset, making migrant students undervalue themselves and have little self-esteem and it also produces difficulties for students to make some accomplishments. According to a study, the mental state of inferiority complex can undermine students' motivation, making them afraid to express themselves and even stop developing themselves [10].

\subsubsection{Loneliness}

In addition to an inferiority complex, another negative mental state of migrant students is loneliness connected with an inferiority complex. A study finds that nearly $40 \%$ of the migrant students are not comfortable when meeting new friends [11]. Sun et al. also argue that one crucial reason to explain why migrant students do not participate actively in class is that they are always worried about whether their words and expressions are proper [11]. Also, cooperating with their classmates in extra-curriculum is a difficult thing to do for migrant students [11]

Under the influence of the inferiority complex, it can be hard for migrant students to have some basic communications with local students, thereby reinforcing the difficulties for them to integrate into the local circles. Hence, they can't get a sense of belonging to the local community and may have feelings of loneliness in their inner world. According to a survey, the tendency for migrant students to feel lonely is stronger than that for local students [13]. $\mathrm{Hu}$ et al. find that some migrant students think that they do not have close friends [13]. What is also shown by the survey is that migrant students have less passion and love for their life [13]. Therefore, it is normal for migrant students to feel that they are not welcomed and excluded in the social circle [11].

\subsubsection{Identification Problem}

Unlike the above two psychological problems, identification problems have more connections with students' identification conditions than emotional states. Therefore, the identification problem would be analyzed in mainly two facets, identity crisis and a sense of resistance to their hometowns.

In terms of an identity crisis, it is suggested that migrant students are confused about what kind of students they are supposed to be seen as, urban students versus rural students. A study finds that $11.2 \%$ of the migrant students think that they are urban students, $18.9 \%$ of them suppose that they are still rural students, and about $69.9 \%$ do not hold clear opinions on their social identity [14].

In migrant students' inner world, there are several reasons to support that they are still rural students. Firstly, migrant students are conscious that they were born in the less developed areas, not in the cities, which is a strong evidence that they are not urban students. In addition, with comparisons made in their mind, there are many differences between themselves and local students in certain aspects, such as their dressing styles and accents. Thirdly, the discrimination posed on migrant students from the local community, no matter from adults or their peers, indicates to migrant students that they are not accepted to be a part of the urban circle. Also, migrant students are not able to become urban students from local people's perspective. However, apart from these three ideas, some other opinions can result in completely the opposite identifications. At the same time of realizing differences between their urban lives and local students' lives, what migrant students may also notice are a lot of similarities between their urban lives and local students' lives compared to their rural lives backward. Therefore, it is reasonable for them to believe that they are just in a transition period of becoming urban students, and they will eventually become urban students. Furthermore, after the rural-to-urban shift, the impact of urban culture on migrant students is stronger than that of rural culture [2]. Living in cities enables migrant students to get a deeper understanding of urban culture, weakening their memories of the countryside. 
To sum up, migrant students might feel marginalized due to the two opposite views [2]. A position in which they seem to be both rural students and urban students contributes to an identity neither rural nor urban. Hence, migrant students are faced with an identity crisis. The second problem in relation to identity is migrant students' resistance in their minds to where they come from. When being aware of the better living standard and educational resources enjoyed by urban students, migrant students are more willing to accept the urban students' identity. A behaviour of migrant students which reveals this state of mind is the imitation of local people's living habits. A survey finds that some migrant students tend to introduce themselves to others as students in cities and not to tell others directly that they are not born in cities [15]. They may also use the local dialect of cities in their daily life in order to be more like urban students [15]. Xiong argues that this behaviour reflects the migrant students' need to integrate into the local community [15].

\subsection{Summary}

Overall, these two factors have some overlapped sections since the mental conditions of migrant students are based on students' own identity and have something to do with the external factors. To illustrate, identification problems come very naturally in students' minds in their urban life. However, discrimination imposed on them also acts as a crucial factor contributing to the idea that they are still students from other areas since they can be conscious of being not accepted by the local circle.

\subsection{Research Gap and Future Direction}

In this field, the previous papers are mainly centered on the disadvantages faced by migrant students. However, these investigations have paid less attention on the benefits to migrant students brought by the migrant lifestyle, which is considered a research gap. Also, there is an obvious absence of sufficient solutions in light of all these problems. Hence, there is expected to be more papers related to these two aspects in this field in the future.

\section{CONCLUSION}

To conclude, this paper's topic is to study the inferior position of migrant students in two factors by analyzing the distinct causes and the effects. Firstly, this paper reviews that discrimination, low quality of migrant schools, and the lack of support from parents all have a negative impact on migrant students' development. In the second place, taking the inner world of migrant students as the study subject, this paper reviews that students are likely to produce three negative mental states, inferiority complex, loneliness, and identification problem. Thus, this paper illustrates the negative situations of migrant students but neglects the positive influence of urban life on migrant students. Also, this paper provides an overview of the possible negative impact on migrant students without corresponding measures to deal with this position. As a result, researchers should focus more on positive qualities gained by migrant students and propose some possible actions which are supposed to be taken by governments, urban citizens, migrant parents, and migrant students themselves in the future.

\section{REFERENCES}

[1] J.L. Shen, X. Liu , J.X. Zhao, B.G. Shi. (2015). The Psychological Development of Chinese Left-Behind Children and Migrant Children in Urbanization Process[J]. Psychological Development and Education, 31(1), 108-116.

[2] M. Xiong, Y.D. Ye. (2011). Mental Health for the Children of Farmers Who Worked in City in China. Advances in psychological science, 19(12), 17981813.

[3] G. Charlotte. (2009). Learning from migrant education: A case study of the schooling of rural migrant children in Beijing. International Journal of Educational Development, 29(5), 495-504.

[4] L.H. Xie. (2015). A Study on the Learning Quality of Migrant Workers' Children in Primary Schools[J]. Journal of Guangxi Teachers Education University (Philosophy and Social Sciences Edition), 51(3), 143-148.

[5] L.B. Liu. (2020). A Study on the Influence of Music Education on Students 'growth. Zhiku Era.

[6] F. Tian. (2010). A Study on the Income Gap between Urban Workers and Migrant Workers. Sociological Studies, 2, 87-105.

[7] W.J. Luan, H.H. Lu, Y.L. Tong, D.N. Lu. (2013). The Influence of Family Relationship on the Mental Health of the Migrant Children. Preschool Education Research, 2, 27-36.

[8] L. Liu, M.H. Fu, Z.Y. Fan. (2010). A Study on Family Rearing Pattern and Learning Adaptability of Migrant Children. Journal of Yangtze Normal University.

[9] T. Hu, C. Guo. (2007). Mental Health Status of Primary School Children of Migrant Workers in Chongqing. School Health in China, 28(8), 745-746.

[10] Z. Fang. (2014). Don't let low self-esteem ruin you. Wenyuan, 5.

[11] W.P. Sun, T. Zhou, Y.Q. Guo, L.H. Zhou. (2007). Mental Health Status of Children of Migrant Workers in a Primary School in Chongqing. School Health in China, 28(1), 68-68. 
[12] G.H. He. (2009). Studies on Inferiority Complex of Migrant Children in Socialization.Mental health education in primary and secondary schools, 11, 47.

[13] Hu Ning, Fang Xiaoyi, Lin Xiuyun, \& Liu Yang. (2009). The Mobility and Social Anxiety and the Effect on Loneliness of Migrant Children in Beijing. Applied Psychology, 2, 166-176.

[14] Y. Liu, X.Y. Fang. (2011). A Study on Social Identity of Migrant Children. Journal of the National School of Administration, 3, 61-66.

[15] Y.H. Xiong. (2009). Urbanized Children: ruralurban cognition and identity consciousness of children of migrant workers. China Rural Watch, 2, 2-11. 